\title{
Etude par simulation Monte Carlo des effets induits dans un TEGFET par le transfert spatial
}

\author{
M. Mouis, P. Dollfus, B. Mougel, J.-F. Pône et R. Castagné \\ Institut d'Electronique Fondamentale, CNRS UA 22, Bât. 220, Université Paris Sud, 91405 Orsay Cedex, \\ France
}

(Reçu le 16 janvier 1987, révisé le 21 mai 1987, accepté le 5 juin 1987)

\begin{abstract}
Résumé. - La simulation de dispositifs TEGFETs a permis d'éclaircir l'influence du transfert spatial sur le mécanisme de saturation du courant de drain dans ce type de transistor. Dans le TEGFET à grille longue $(1 \mu \mathrm{m})$, ce phénomène n'apparaît pas et le courant de drain est saturé par pincement du canal. Dans le TEGFET à grille courte $(0,5 \mu \mathrm{m})$, à la fois le transfert spatial et le transfert intervallées interviennent, mais sans être directement la cause de la saturation. Néanmoins le transfert spatial empêche la formation d'un domaine dipolaire ; le courant de drain est alors contrôlé par le rétrécissement du canal sous la grille. Les électrons défocalisés restent sous l'influence de la tension de drain, provoquant ainsi une forte conductance de drain. On remarque également que le transfert spatial répartit le courant dans les différentes couches conductrices sans modifier le courant de drain total. Dans un dispositif à deux drains - l'un collectant les électrons du canal, l'autre les électrons transférés - le transfert spatial permet d'obtenir une résistance différentielle négative.
\end{abstract}

\begin{abstract}
Simulations of TEGFET devices explain the influence of real space transfer on the saturation mechanism of the drain current in this transistor. In a long gate device $(1 \mu \mathrm{m})$, this transfer is not observed and the drain current is saturated by channel pinch-off. In a short gate TEGFET $(0.5 \mu \mathrm{m})$, both real space transfer and $k$-space transfer occur without being directly responsible for saturation. Real space transfer nevertheless precludes the formation of a dipolar domain; the drain current is then controlled by channel narrowing under the gate. The spread electrons stay under control of the drain contact and a high drain conductance can be observed. We also note that real space transfer distributes the current between the various conducting layers without changing the total drain current. In a two drain contacts device - one contact collecting the channel electrons and the other the transferred electrons - the real space transfer allows a negative differential resistance to be obtained.
\end{abstract}

\section{Introduction.}

Dans un TEGFET, les électrons circulent dans la couche d'accumulation confinée contre l'hétéro-barrière interfaciale. Si le semi-conducteur à grand gap peut être considéré comme un isolant, le comportement du dispositif est similaire à celui d'un MOSFET. Cependant, la technologie utilisée pour faire croître cette couche permet d'obtenir une meilleure qualité d'interface que dans le transistor MOS classique. De plus, la faible longueur de canal des TEGFETs favorise les phénomènes de transport non-stationnaires. Lorsqu'ils sont chauffés par le champ électrique, les porteurs peuvent acquérir une énergie cinétique suffisante pour franchir l'hétérojonction (environ $0,3 \mathrm{eV}$ leur sont nécessaires). Ce phénomène est appelé transfert spatial [1].
Ce phénomène dépend à la fois du chauffage des électrons et de la dispersion de leur vecteur d'onde dans la direction normale à l'interface ; il est donc difficile à prendre en compte de façon précise dans des modèles de type hydrodynamique [2]. Une description détaillée de la structure de bande de chaque semi-conducteur et des interactions électron-phonon est alors nécessaire à la compréhension du comportement du dispositif en régime de saturation. Pour obtenir cette description dans un système unique, nous avons étudié le transfert dans l'espace réel et ses conséquences sur le comportement d'un TEGFET GaAs/GaAlAs en utilisant un modèle de type particulaire Monte Carlo prenant en compte l'effet de la répartition des porteurs sur le champ électrique [3]. Ce modèle résoud l'équation de Poisson à deux dimensions dans l'espace géométrique et 
simule le mouvement des porteurs sous l'effet du champ électrique et des intéractions avec les imperfections du réseau (phonons inter-vallées et intravallée, impuretés et désordre d'alliage). Les vallées $\Gamma, \mathrm{L}$ et $\mathrm{X}$ sont considérées comme étant sphériques non paraboliques.

Le dispositif étant étudié à température ambiante, les effets du confinement bidimensionnel dans le puits interfacial tant sur la structure de bande des électrons que sur les phonons ou sur les interactions électrons/réseau ne sont pas prises en 'compte. Le mouvement des électrons est donc simulé, comme en volume, dans les trois dimensions de l'espace des vecteurs d'onde. Cette approximation est justifiée dans la zone de saturation du dispositif où les électrons ont une grande énergie cinétique et où le confinement est faible [4]. Dans les zones d'accès, elle est valable dans la mesure où le dispositif comporte une couche GaAlAs relativement peu dopée et où on étudie son fonctionnement à température ambiante. Par contre, à basse température [5] ou pour des dopages plus élevés de GaAlAs [6], le caractère bidimensionnel du gaz d'électrons dans les zones d'accès peut influer indirectement sur le transfert spatial en modifiant la répartition en énergie et en vecteur d'onde des électrons à l'entrée de la zone de transfert.

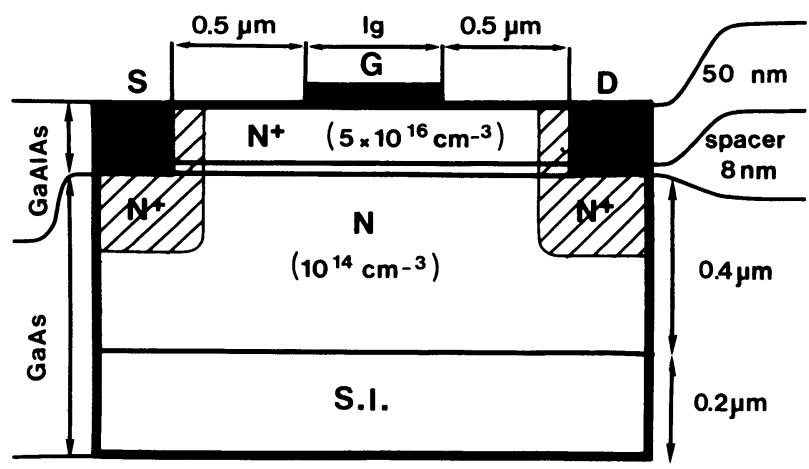

Fig. 1. - Paramètres géométriques et niveaux de dopage des TEGFETs simulés. $l_{\mathrm{g}}=0,5$ ou $1 \mu \mathrm{m}$.

[Geometrical parameters and doping levels of simulated TEGFETs. $l_{\mathrm{g}}=0.5$ or $1 \mu \mathrm{m}$.]

Les TEGFETs GaAs/GaAlAs simulés ont des dimensions géométriques classiques (Fig. 1). On peut remarquer que les contacts de source et de drain sont ohmiques à la fois sur le GaAlAs et sur le GaAs. Ceci correspond à la situation où les contacts sont pris sur le GaAs à travers la couche de GaAlAs. Le transfert spatial et le mécanisme de saturation ont été étudiés en faisant varier des paramètres significatifs de la géométrie des différents dispositifs simulés.

\section{TEGFET à grille longue.}

Le dispositif simulé est un TEGFET ayant une grille de $1 \mu \mathrm{m}$ de long. La distribution spatiale des électrons en régime de saturation dans les vallées $\Gamma$, L et $\mathrm{X}$ est représentée sur la figure 2 . Une région à fort champ électrique $(>10 \mathrm{kV} / \mathrm{cm})$ est présente à la fin de la grille sur une distance d'environ $0,3 \mu \mathrm{m}$. En raison du phénomène de survitesse, l'énergie cinétique moyenne des porteurs reste relativement faible, de telle sorte que, ni le transfert intervallées, ni le transfert spatial ne sont significatifs. D'autre part, le champ électrique transverse s'inverse et devient

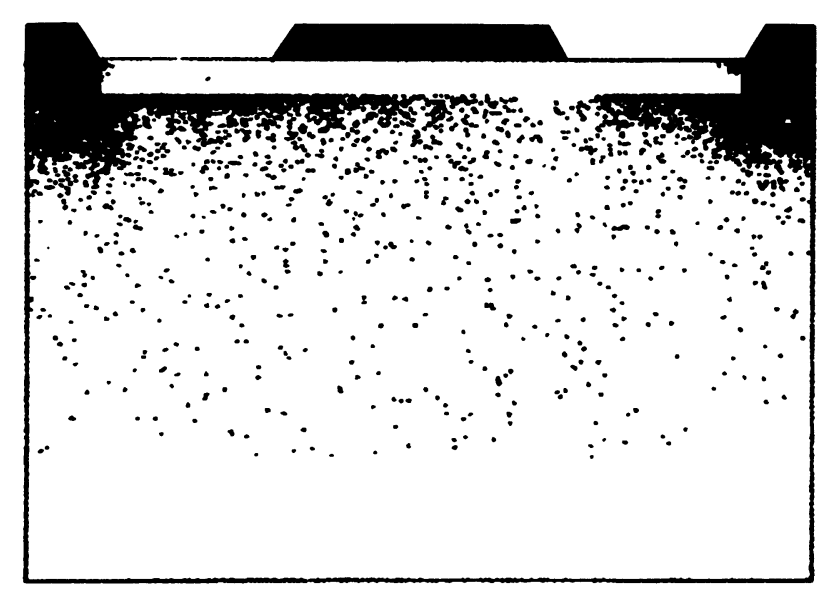

PARTICULES

DANS GAMIA a)

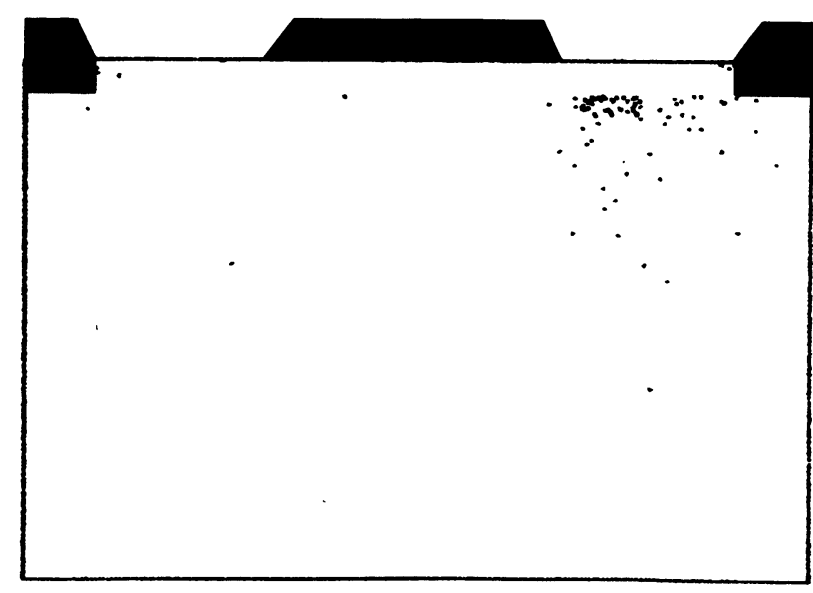

PARTICULES

DANS $L, X$

b)

Fig. 2. - Distribution spatiale des électrons dans le TEGFET de la figure 1 pour une longueur de grille de $1 \mu \mathrm{m}$; (a) électrons dans la vallée $\Gamma$ (b) électrons dans les vallées $\mathrm{L}$ et $\mathrm{X}$ pour des tensions $V_{\mathrm{gs}}=-0,2 \mathrm{~V}+\Phi_{\mathrm{B}}$, $V_{\mathrm{ds}}=0,5 \mathrm{~V}$, où le courant de drain est saturé.

[Spatial distribution of electrons in the $1 \mu \mathrm{m}$ gate length TEGFET on figure 1: (a) electrons in the $\Gamma$ valley (b) electrons in the $\mathrm{L}$ and $\mathrm{X}$ valleys with bias voltages in the drain current saturation region: $V_{\mathrm{gs}}=-0.2 \mathrm{~V}+$ $\left.\Phi_{\mathrm{B}}, V_{\mathrm{ds}}=0.5 \mathrm{~V}.\right]$ 
négatif, donnant lieu au pincement du canal et à sa défocalisation. La saturation du courant de drain est donc liée au pincement du canal. Les évolutions de la conductance de drain et de la position du point de pincement sont bien prédites par un modèle classique simple de transistor MOS.

\section{TEGFET à grille courte.}

La situation dans un dispositif de $0,5 \mu \mathrm{m}$ de longueur de grille est différente : à la fois le transfert dans l'espace réciproque et le transfert spatial sont présents au voisinage du drain quand le courant dans le dispositif est saturé. Le problème est alors de déterminer le mécanisme responsable de cette saturation :

- le pincement classique du canal ;

- le transfert dans l'espace réciproque, qui crée une charge d'espace écrantant le potentiel de drain ;

- le transfert spatial vers la couche de GaAlAs, où la conduction électronique est moins efficace.

Afin de comprendre si ce transfert spatial est responsable de la saturation, nous avons simulé des dispositifs identiques en changeant artificiellement la hauteur de barrière de l'hétérojonction. Les distributions de porteurs pour des hauteurs de barrière de $0,25,0,3$ et $0,5 \mathrm{eV}$ sont représentées sur la figure 3 . Bien que ces distributions soient très différentes (la concentration en électrons étant plus grande dans le GaAlAs quand la barrière est moins haute), les courants de drain sont les mêmes dans les trois cas (Fig. 4). Nous pouvons donc dire que le transfert spatial n'est pas directement responsable de la saturation du courant.

L'énergie moyenne des électrons est tracée sur la figure 5 en fonction de la position sous la grille, pour différentes tensions drain-source. Une portion significative des porteurs n'est transférée dans les vallées latérales (l'énergie moyenne devient de l'ordre du gap intervallées) que lorsque $V_{\mathrm{Ds}}$ atteint environ $0,5 \mathrm{~V}$, alors que le courant de drain est saturé dès que $V_{\text {DS }}$ atteint $0,25 \mathrm{~V}$. Nous ne pouvons donc pas dire que le transfert dans les vallées latérales induit la saturation. Quand le transfert intervallées devient efficace, l'isotropie des interactions électron-phonon dans les vallées $L$ peut favoriser l'orientation $\mathrm{du}$ vecteur d'onde dans la direction normale à l'interface, augmentant ainsi la probabilité de transfert

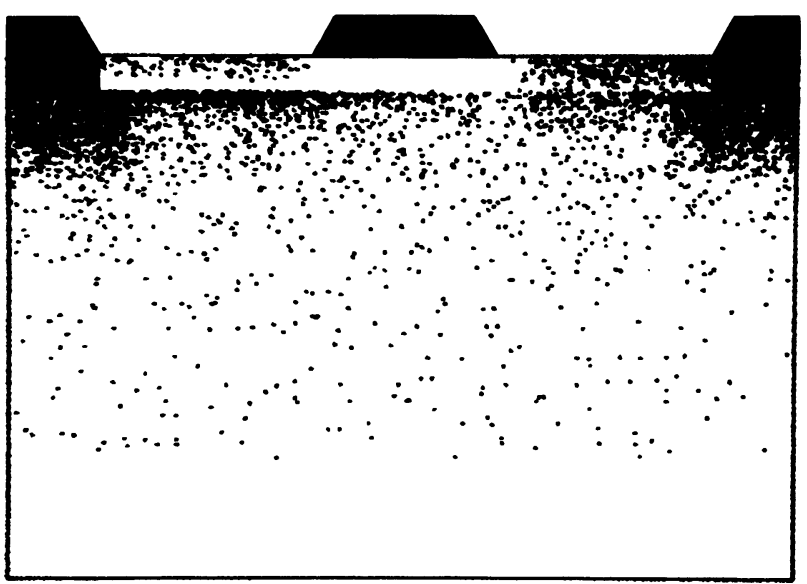

a)

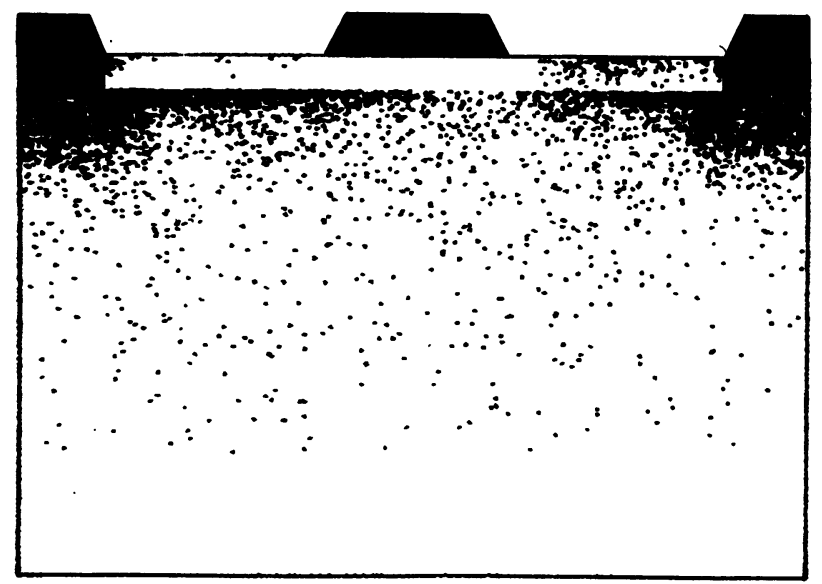

b)

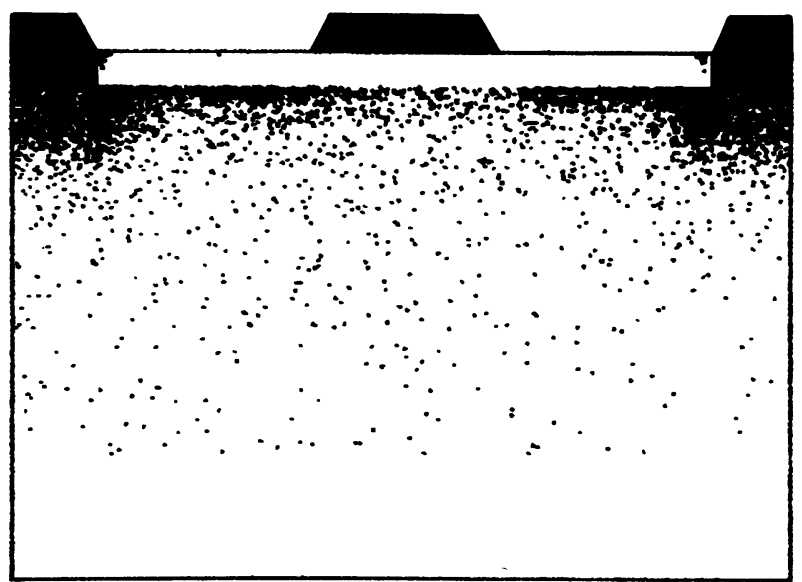

PARTICULES

c)

Fig. 3. - Distribution spatiale des électrons dans le TEGFET de la figure 1 avec une longueur de grille de $0,5 \mu \mathrm{m}$ pour différentes valeurs de la hauteur de barrière de l'hétérojonction $\Delta E_{\mathrm{c}}$ et des tensions de polarisation identiques à la variation de tension de seuil avec $\Delta E_{\mathrm{c}}$ près :
(a) $\Delta E_{\mathrm{c}}=0,25 \mathrm{eV}, V_{\mathrm{gs}}=-0,15 \mathrm{~V}+\Phi_{\mathrm{B}}, V_{\mathrm{ds}}=0,5 \mathrm{~V}$;
(b) $\Delta E_{\mathrm{c}}=0,3 \mathrm{eV}, V_{\mathrm{gs}}=-0,2 \mathrm{~V}+\Phi_{\mathrm{B}}, V_{\mathrm{ds}}=0,5 \mathrm{~V}$;
(c) $\Delta E_{\mathrm{c}}=0,5 \mathrm{eV}, V_{\mathrm{gs}}=-0,4 \mathrm{~V}+\Phi_{\mathrm{B}}, V_{\mathrm{ds}}=0,5 \mathrm{~V}$.

[Spatial distribution of electrons in the $0.5 \mu \mathrm{m}$ gate length TEGFET of figure 1 for different values of the heterojunction barrier height $\Delta E_{\mathrm{c}}$ and the same bias voltage apart from a difference in the threshold voltage due to the $\Delta E_{\mathrm{c}}$ variation :
(a) $\Delta E_{\mathrm{c}}=0.25 \mathrm{eV}, V_{\mathrm{gs}}=-0.15 \mathrm{~V}+\Phi_{\mathrm{B}}, V_{\mathrm{ds}}=0.5 \mathrm{~V}$;
(b) $\Delta E_{\mathrm{c}}=0.3 \mathrm{eV}, V_{\mathrm{gs}}=-0.2 \mathrm{~V}+\Phi_{\mathrm{B}}, V_{\mathrm{ds}}=0.5 \mathrm{~V}$;
(c) $\Delta E_{\mathrm{c}}=0.5 \mathrm{eV}, V_{\mathrm{gs}}=-0.4 \mathrm{~V}+\Phi_{\mathrm{B}}, V_{\mathrm{ds}}=0.5 \mathrm{~V}$.] 


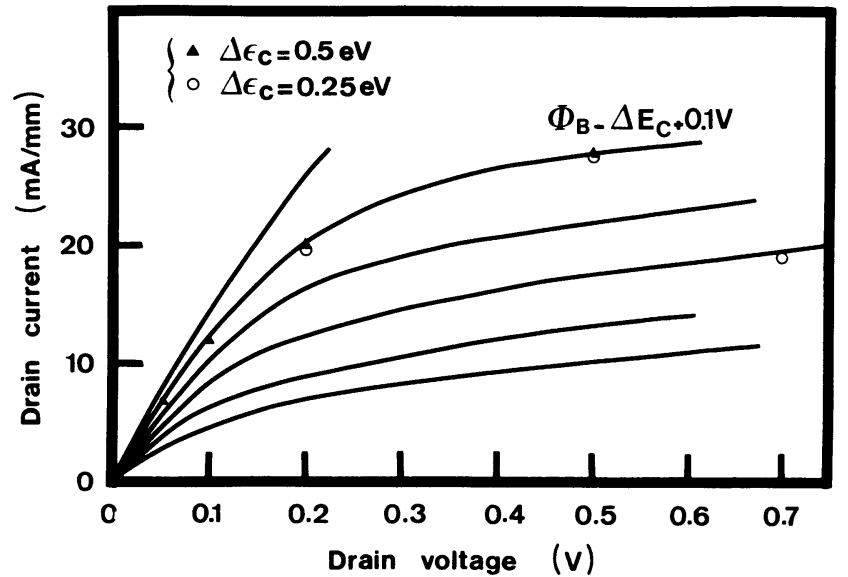

Fig. 4. - Caractéristiques de drain pour différentes valeurs de la hauteur de barrière ; les traits pleins correspondent à $0,3 \mathrm{eV}$.

[Drain characteristics for different values of the barrier height ; full lines are for $0.3 \mathrm{eV}$.]

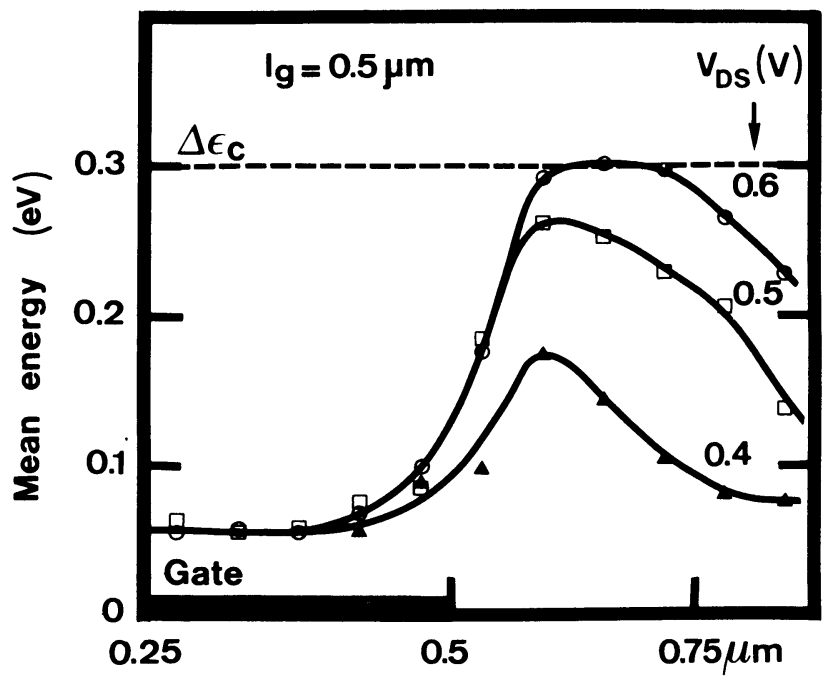

Fig. 5. - Energie moyenne des porteurs dans le canal sous la grille en fonction de la position, pour différentes tensions de drain.

[Mean energy of the carriers in the channel under the gate as a function of position, for different drain biases.]

spatial. Ceci peut être observé sur la figure 5 , où l'énergie moyenne sature à une valeur correspondant à la hauteur de barrière. Le transfert spatial empêche ensuite la formation d'un domaine en défocalisant les électrons dans la couche de GaAlAs.

Ainsi, plusieurs phénomènes de défocalisation des porteurs empêchent la prise de contrôle du courant par un domaine dipolaire. Le courant de drain est alors contrôlé par le rétrécissement du canal sous la grille, et l'écrantement du potentiel de drain reste faible. Ceci conduit à une grande valeur de la conductance de drain.

\section{Influence du contact de drain.}

Dans le cas précédent, le transfert spatial donnait lieu à un courant de fuite à travers la couche de GaAlAs. Le courant drain collecté sur chaque couche est tracé sur le figure 6 . Si le contact est pris sur le GaAs uniquement (en utilisant une structure mesa par exemple), la charge stockée dans le GaAlAs est plus importante, mais le courant drain reste identique (les électrons reviennent dans le GaAs et contribuent au courant total). Ceci est cohérent avec les résultats précédents, car la distribution des porteurs sous la grille (qui contrôle le courant) n'est pas perturbée.

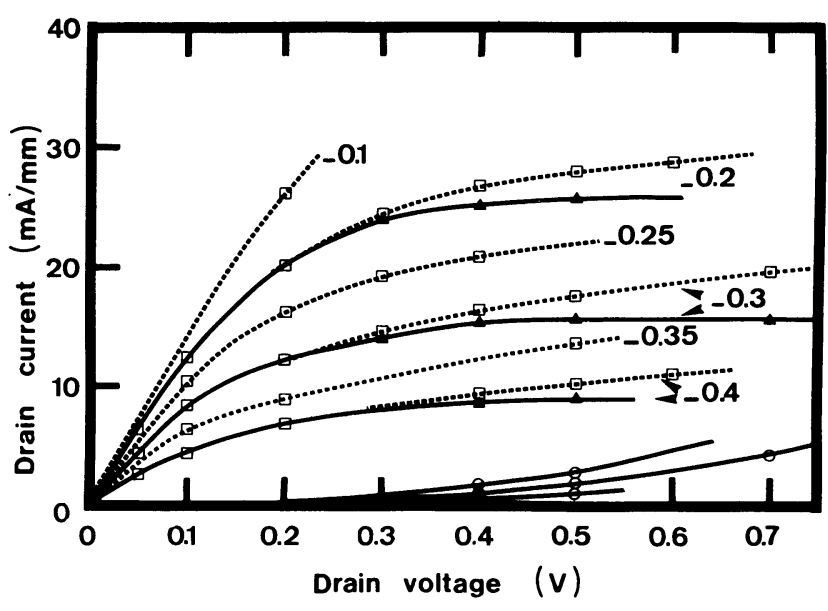

Fig. 6. - Caractéristiques de drain du TEGFET de $0,5 \mu \mathrm{m}$ de longueur de grille ; carrés : courant drain total ; triangles : courant collecté sur le GaAs ; cercles : courant collecté sur le GaAlAs.

[Drain characteristics of the $0.5 \mu \mathrm{m}$ gate length TEGFET ; squares : total drain current ; triangles : current collected on GaAs ; circles : current collected on GaAlAs.]

On peut toutefois remarquer que si le courant circulant dans le GaAlAs pouvait être collecté par une autre électrode, le courant drain circulant dans le $\mathrm{GaAs}$ pourrait être très bien saturé, et même montrer une résistance différentielle négative.

\section{Utilisation du transfert spatial dans un dispositif à résistance différentielle négative.}

Pour évaluer la possibilité de résistance différentielle négative (RDN) qui peut être obtenue de cette façon, nous avons simulé un dispositif ayant deux contacts de drain, l'un sur la couche de GaAs $\left(D_{1}\right)$ et l'autre sur le GaAlAs $\left(D_{2}\right)$. Ce dispositif est représenté sur la figure 7 . Ses caractéristiques de drain et la distribution spatiale des porteurs (Fig. 8) montrent une résistance différentielle négative pour des courants drain normaux, lorsque le transfert spatial des électrons du GaAs vers le GaAlAs 


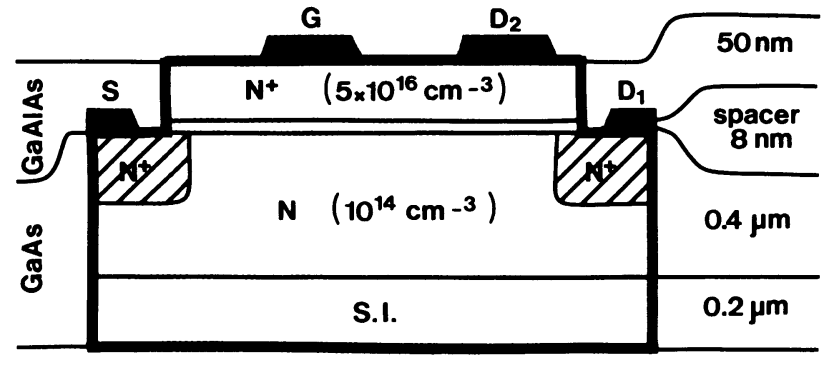

Fig. 7. - Dispositif à deux contacts de drain.

[Device with two drain contacts.]

ID $(\mathrm{mA} / \mathrm{mm})$

$v_{D 1}(v)$

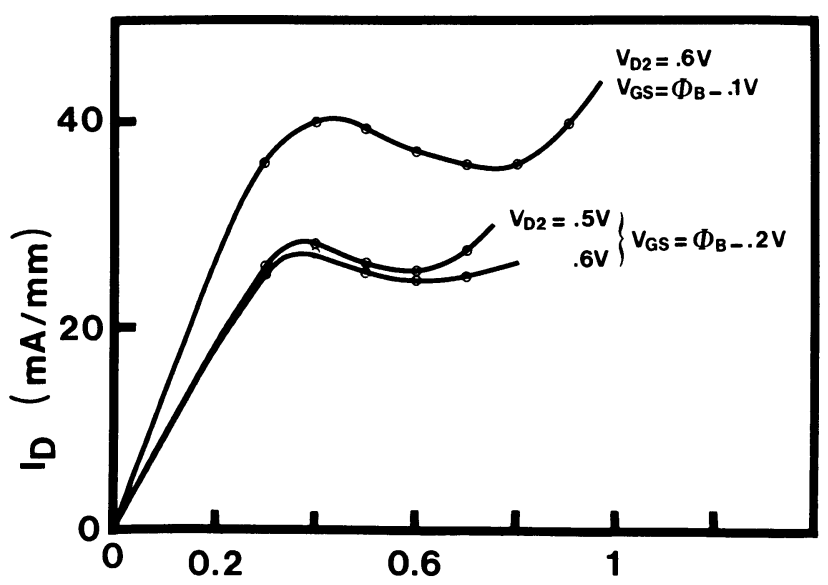

a)

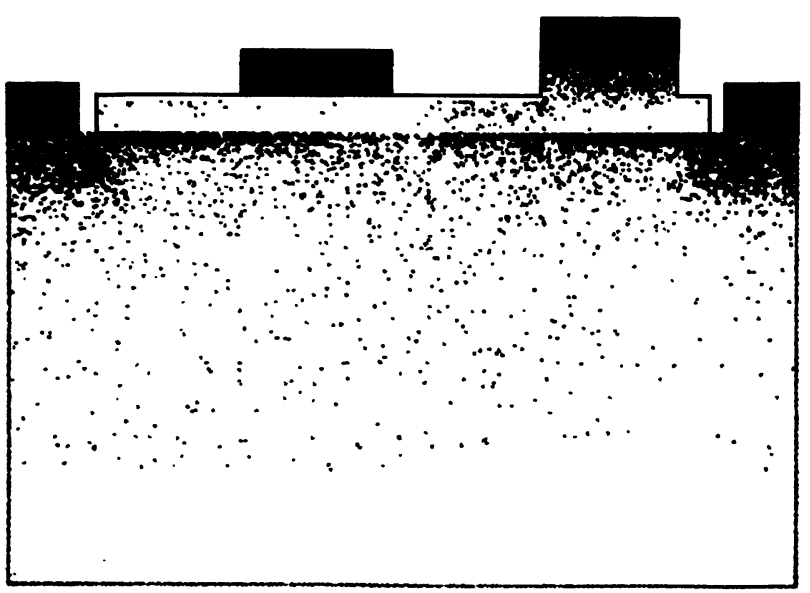

c)

Fig. 8. - Caractéristiques de drain (a) et distributions de porteurs dans le dispositif à deux drains de la figure 7 pour les tensions de polarisations $V_{\mathrm{gs}}=-0,1 \mathrm{~V}+\Phi_{\mathrm{B}}, V_{\mathrm{d} 2}=$ $0,6 \mathrm{~V}, V_{\mathrm{d} 1}=0,3 \mathrm{~V}(\mathrm{~b}) ; 0,6 \mathrm{~V}(\mathrm{c}) ; 0,9 \mathrm{~V}(\mathrm{~d})$. apparaît. Cependant, la partie de la caractéristique présentant cette RDN ne peut pas être très importante, car le transfert spatial ne concerne qu'une portion de la population.

\section{Conclusion.}

La saturation du courant de drain du dispositif à grille courte étudié ici est contrôlée par le rétrécissement du canal sous la grille. Le transfert spatial n'a pas d'influence sur le courant de drain total mais peut changer sa répartition entre les différentes couches conductrices. Cet effet peut être utilisé pour dimensionner des dispositifs présentant une résistance différentielle négative.

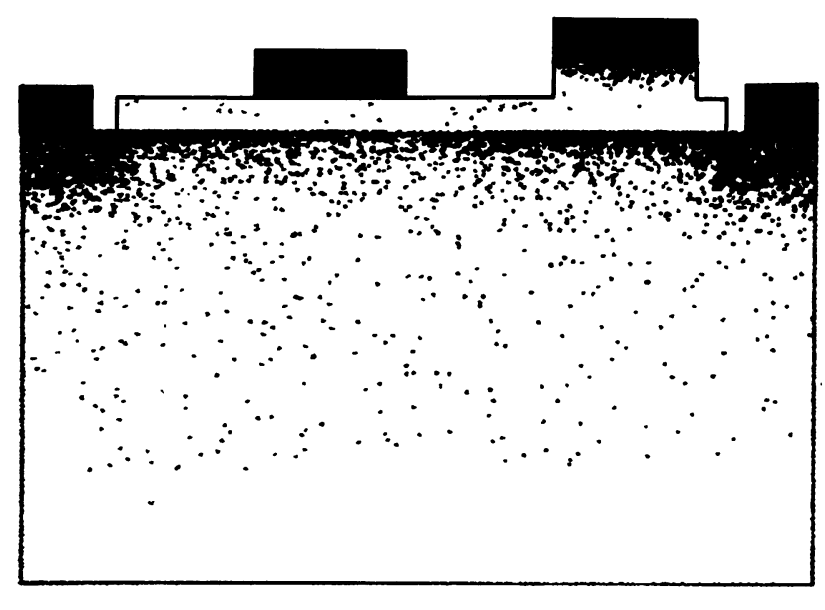

b)

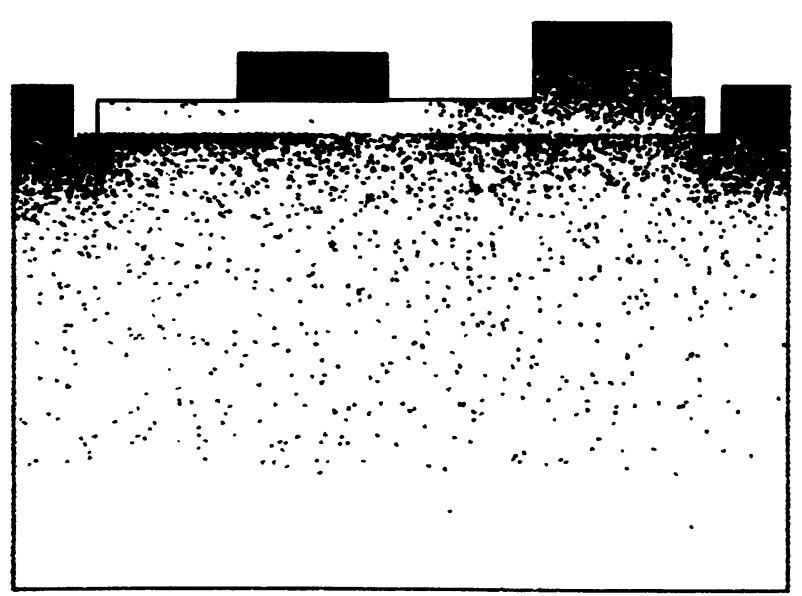

d)

[Drain characteristics (a) and particle distributions in the two-drain device of figure 7 for bias voltages : $V_{\mathrm{gs}}=$ $-0.1 \mathrm{~V}+\Phi_{\mathrm{B}}, V_{\mathrm{d} 2}=0.6 \mathrm{~V}, V_{\mathrm{d} 1}=0.3 \mathrm{~V}(\mathrm{~b}) ; 0.6 \mathrm{~V}(\mathrm{c})$; $0.9 \mathrm{~V}(\mathrm{~d})$. 


\section{Bibliographie}

[1] Voir par exemple: Hess, K., in proceedings of ICPS 16, Montpellier (North Holland) 1982, 723.

[2] Widiger, D., Hess, K., Coleman, J. J., I.E.E.E. Electron Device Lett. EDL-5(7) (1984) 266-269.

[3] Hesto, P., Pône, J. F., Mouis, M., Pelouard, J.L., CASTAgné, R., in proceedings of NASE-
CODE IV Conference, Dublin (Boole Press, Dublin) 1985.

[4] Ravaioli, U., Ferry, D. K., I.E.E.E. Trans. Electron Devices, ED-33(5) (1986) 677.

[5] Bastard, G., Proceedings of the 5th Int. Conf. Electronic Properties of Two Dimensional Systems (Oxford), Surf. Sci. 142 (1983) 284.

[6] Vinter, B., Appl. Phys. Lett. 44 (1984) 308. 